\title{
Ramificação e distribuição dos nervos frênicos no músculo diafragma do gato doméstico
}

Pamela Rodrigues Reina MOREIRA ${ }^{1}$

Gregório Corrêa GUIMARÃES²

Márcia Rita Fernandes

$\mathrm{MACHADO}^{2}$

André Luiz Quagliatto

SANTOS ${ }^{3}$

Sílvia Helena Brendolan

GERBASI $^{4}$

Maria Angélica MIGLINO ${ }^{5}$

\section{Correspondência para:}

Departamento de Morfologia e Fisiologia Animal da FCAV/UNESP. Via de Acesso Professor Paulo Donato Castellane, s/n. 14884-900. Jaboticabal SP. E-mail: gregorio@fcav.unesp.br

Recebido para publicação: 10/08/2005 Aprovado para publicação: 27/03/2007

\author{
1Faculdade de Ciências Agrárias, Universidade de Marília, Marília - SP \\ ${ }^{2}$ Faculdade de Ciências Agrárias e Veterinárias, Universidade Estadual Paulista, \\ Jaboticabal - SP \\ ${ }^{3}$ Faculdade de Medicina Veterinária da Universidade Federal de Uberlândia - MG \\ ${ }^{4}$ Centro Universitário Barão de Mauá, Ribeirão Preto - SP \\ ${ }^{5}$ Faculdade de Medicina Veterinária e Zootecnia da Universidade de São Paulo - SP
}

\section{Resumo}

Palavras-chave: Ramificação.

A ramificação e a distribuição dos nervos frênicos direito e esquerdo foram estudadas em 30 músculos diafragmas de gatos domésticos sem raça definida, 7 machos e 23 fêmeas, conservados em solução de formaldeído a $10 \%$. Mediante fixação e dissecção, observou-se que os nervos frênicos se ramificaram para as respectivas partes carnosas do músculo diafragma, pars lumbalis, costalis e sternalis, e terminaram em maior frequêencia em tronco dorsolateral e ramo ventral $(63,33 \%)$. Notou-se ainda os seguintes arranjos: ramos dorsal, lateral e ventral (25,0 \%); ramo dorsal e tronco ventrolateral $(6,66 \%)$; troncos dorsolateral e ventrolateral (3,33\%); tronco dorsolateral, ramos lateral e ventral (1,66\%). Os nervos frênicos se distribuíram simetricamente em 11 exemplares (36,66\%), exibindo somente a terminação em tronco dorsolateral e ramo ventral. Os ramos dorsais inervaram a pars lumbalis $(73,33 \%$ à direita e 56,66 \% à esquerda) e a pars costalis (13,33\% à direita e 10,0\% à esquerda). $\mathrm{O}$ ramo dorsal direito supriu a crus mediale dexter do pilar direito $(100,0$ $\%$ ) e o ramo dorsal esquerdo inervou a crus mediale sinister do pilar direito e o pilar esquerdo (100,0 \%). Os ramos laterais inervaram a pars lumbalis (23,33\% à direita e 33,33 \% à esquerda), a pars costalis (96,66 \% à direita e 100,0 \% à esquerda) e a pars sternalis $(3,33 \%$ somente à direita). Os ramos ventrais inervaram a região ventral da pars costalis (46,66 \% à direita e 43,33\% à esquerda) e a pars sternalis (96,66 \% à direita e 100,0 \% à esquerda). Quatro animais fêmeas $(13,33 \%)$ exibiram cruzamento de fibras provenientes do ramo ventral esquerdo para o antímero direito sendo que em um desses exemplares $(3,33 \%)$ ocorreu conexão entre o ramo ventral esquerdo e o direito.

\section{Introdução}

Encontrado somente nos mamíferos e considerado o principal músculo da respiração, o diafragma, se apresenta sob a forma de uma cúpula convexa em sua superfície cranial, determinando o limite caudal da cavidade torácica e o cranial da cavidade abdominal ${ }^{1,2}$. Este músculo tem como principal supridor o nervo frênico, que foi examinado em diferentes espécies animais ${ }^{3}$.
Os ramos nervosos formadores dos nervos frênicos unem-se em diferentes níveis, sob a $1^{\mathrm{a}}, 2^{\mathrm{a}}, 3^{\mathrm{a}}$ ou $4^{\mathrm{a}}$ costelas ou espaços intercostais correspondentes, após abandonarem as respectivas raízes cervicais ${ }^{4,5}$.

Cada um dos nervos frênicos pouco antes de atingir o músculo diafragma dividese em três ramos terminais: dorsal, lateral e ventral. Estes se dirigem, respectivamente, à parte lombar (pars lumbalis), parte costal (pars costalis) e parte esternal (pars sternalis) da 
porção carnosa do músculo diafragma ${ }^{1,2,6}$.

Nos carnívoros, os nervos frênicos se ramificam no músculo diafragma, para o qual eles são a única inervação motora, cujas fibras sensitivas são suplementadas por ramos emitidos pelos nervos intercostais ${ }^{6}$.

Ao penetrar no músculo diafragma do cão, segundo Merendino et al. ${ }^{7}$, o nervo frênico divide-se em quatro ramos terminais: ventral, ventrolateral, dorsolateral e dorsal, que se arranjam à semelhança de uma algema. Como o trajeto de ambos os nervos frênicos, direito e esquerdo são semelhantes, sua distribuição pode ser considerada um exemplo de dupla algema, entretanto, podem ocorrer variações. Os ramos ventrolateral e dorsolateral são os maiores, seguem um curso mais longo e inervam a maior parte do músculo diafragma ${ }^{7}$.

Conde ${ }^{8}$ descreveu nos suínos que o nervo frênico terminava tanto à direita quanto à esquerda, em ramos dorsal e tronco ventrolateral, e raramente, em ramos ventral e tronco dorsolateral. Os troncos dorsolateral e ventrolateral, após curto trajeto, dividiamse em ramos dorsal e lateral e em ventral e lateral, resultando segundo o autor, na forma habitual de ramificação dos nervos frênicos, correspondente a disposição dos três ramos diafragmáticos fundamentais: um dorsal, um lateral e um ventral.

Amorim Júnior, Bittencourt e Amorim ${ }^{9}$ observaram no sagüi que os ramos dorsais dos nervos frênicos distribuíam-se sempre a pars lumbalis tanto à direita como à esquerda, os ramos laterais inervavam sempre a pars costalis do antímero correspondente, $\mathrm{e}$ os ramos ventrais, distribuíam-se sempre a pars sternalis e a pars costalis do respectivo antímero.

Descrições específicas sobre a ramificação e distribuição do nervo frênico na espécie felina não foram encontradas na literatura consultada, assim, buscou-se neste trabalho conhecer a ramificação e a distribuição do nervo frênico no músculo diafragma do gato doméstico, com finalidade de fornecer informações específicas de sua apresentação nesta espécie.

\section{Material e Método}

Foram utilizados 30 músculos diafragmas de gatos adultos, sem raça definida, sete machos e 23 fêmeas, animais estes já utilizados em experimentos conduzidos anteriormente no Laboratório de Anatomia da FCAV/UNESP.

Os músculos diafragmas foram isolados e acondicionados em um recipiente contendo solução aquosa de formaldeído a $10 \%$. Cada músculo diafragma foi abordado em sua face cranial, sendo necessário para a dissecção dos nervos frênicos uma lupa com aumento de 10 vezes, além de pinças anatômicas e tesouras.

A nomenclatura adotada para este estudo esteve de acordo com o International Committee on Veterinary Gross Anatomical Nomenclature ${ }^{10}$.

\section{Resultados}

Os nervos frênicos se ramificaram no músculo diafragma em maior freqüência em tronco dorsolateral e ramo ventral $(63,33 \%)$ seguido da terminação em ramos dorsal, lateral e ventral $(25,0 \%)$. Além dessas ramificações, evidenciou-se terminação em ramo dorsal e tronco ventrolateral $(6,66 \%)$, em troncos dorsolateral e ventrolateral $(3,33$ $\%$ e em tronco dorsolateral, ramo lateral e ramo ventral $(1,66 \%)$, demonstrado na tabela 1 e figuras 1 e 2.

Os nervos frênicos exibiram ramificação simétrica em 11 exemplares $(36,66 \%)$, terminando sempre em tronco dorsolateral e ramo ventral, notado em três machos e oito fêmeas (Figura 2A).

Quanto à distribuição dos ramos dos nervos frênicos no músculo diafragma do gato, observou-se que os ramos dorsais inervaram a pars lumbalis $(73,33 \%$ à direita $\mathrm{e}$ $56,66 \%$ à esquerda) e a pars costalis (13,33\% à direita e 10,0 \% à esquerda). O ramo dorsal direito supriu a crus mediale dexter do pilar direito $(100,0 \%)$ e o ramo dorsal esquerdo inervou a crus mediale sinister do pilar direito e o pilar esquerdo (100,0\%). Os ramos laterais inervaram a pars lumbalis $(23,33 \%$ à direita $\mathrm{e}$ 
Tabela 1 - Ramificação dos nervos frênicos no músculo diafragma do gato doméstico de acordo com o antímero. Faculdade de Ciências Agrárias e Veterinárias, UNESP, Jaboticabal - SP, 2005.

\begin{tabular}{cccccc}
\hline Ramos & \multicolumn{2}{c}{ Antímero } & \multirow{2}{*}{ Total } & (\%) \\
\cline { 2 - 3 } & Direito & Esquerdo & & \\
\hline Dorsolateral e ventral & 23 & 15 & 38 & 63,33 \\
Dorsal, lateral e ventral & 02 & 13 & 15 & 25,0 \\
Dorsal e ventrolateral & 03 & 01 & 04 & 6,66 \\
Dorsolateral e ventrolateral & 02 & 00 & 02 & 3,33 \\
Dorsolateral, lateral e ventral & 00 & 01 & 01 & 1,66 \\
\hline
\end{tabular}
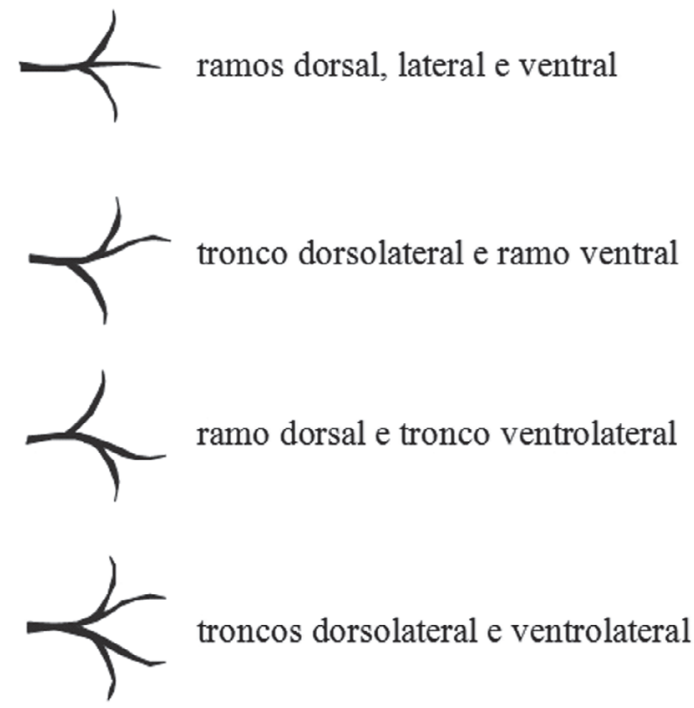

troncos dorsolateral e ventrolateral

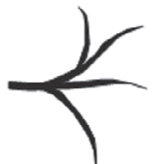

tronco dorsolateral e ramos lateral e ventral

Figura 1 - Ramificações exibidas pelos nervos frênicos no músculo diafragma do gato doméstico

$33,33 \%$ à esquerda), a pars costalis $(96,66 \%$ à direita e 100,0\% à esquerda) e a pars sternalis (3,33\% somente à direita). Os ramos ventrais inervaram a região ventral da pars costalis (46,66\% à direita e $43,33 \%$ à esquerda) e a pars sternalis $(96,66 \%$ à direita e $100,0 \%$ à esquerda), o que pode ser observado na figura $2 \mathrm{~A}-\mathrm{H}$.

Pode-se evidenciar em quatro fêmeas $(13,33 \%)$ o cruzamento de fibras nervosas provenientes do ramo ventral do nervo frênico esquerdo para o antímero direito (Figura 3). Observou-se em um desses casos a ocorrência de conexão entre fibras dos ramos ventrais dos nervos frênicos esquerdo e direito (Figura 3A).

\section{Discussão}

No estudo particularizado dos nervos frênicos do gato doméstico, observou-se em maior freqüência a bifurcação em tronco dorsolateral e ramo ventral, 23 casos à direita $(76,66 \%)$ e 15 casos à esquerda (50,0\%), o que mais se aproxima das observações de Conde ${ }^{11}$ no antímero direito de cães $(85,0 \%)$, de Santiago e Pereira ${ }^{12}$ em eqüinos (96,7 \%), de Miglino, Prada e Souza ${ }^{13}$ em búfalos (53,3 $\%$ e de Amorim Júnior, Bittencourt e Amorim $^{9}$ no sagüi $(46,6 \%$ ). Entretanto Conde $^{8}$ descreveu essa terminação como sendo a mais rara nos suínos, com ocorrência em apenas 1,6\% dos exemplares. 

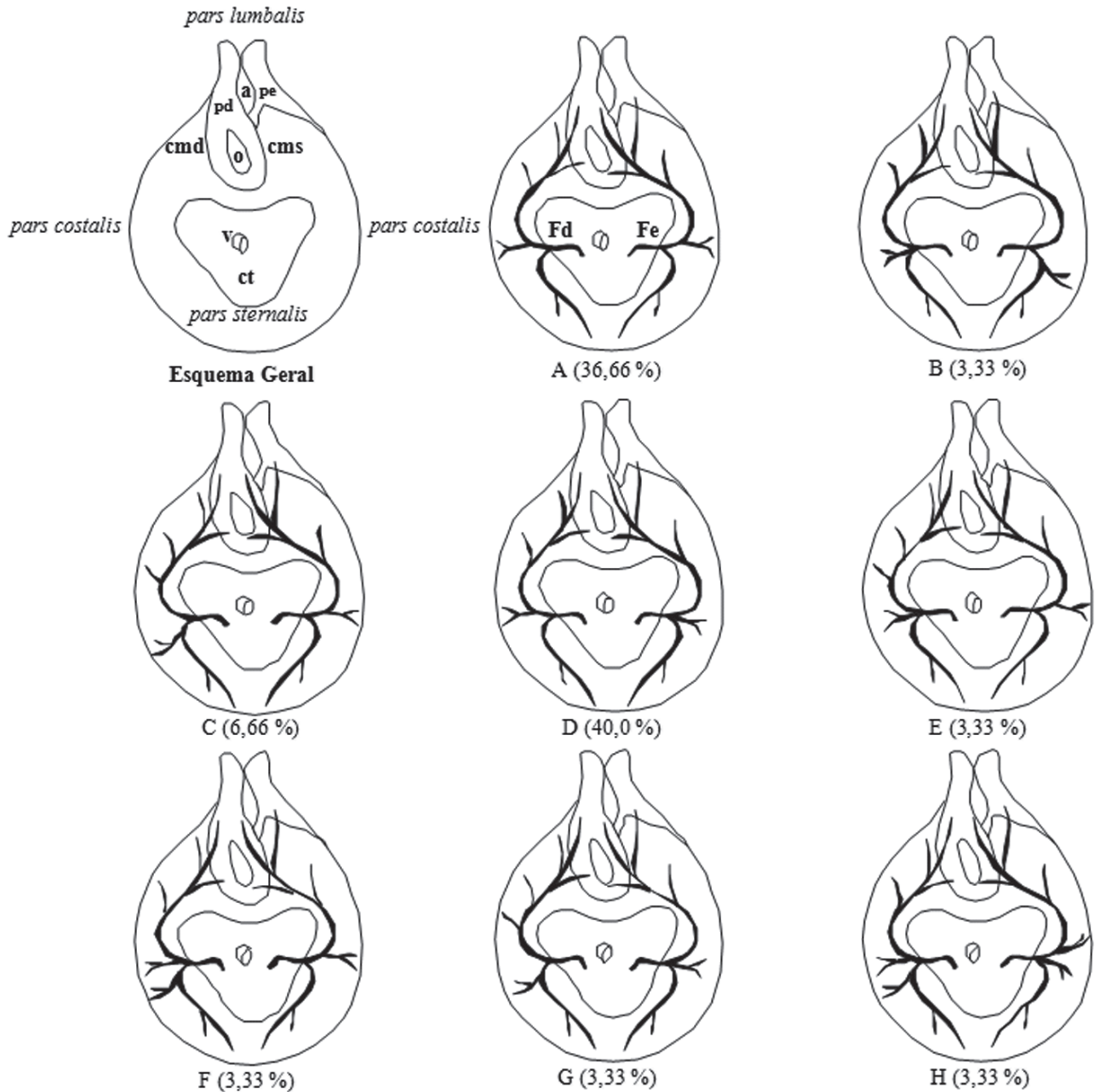

Figura 2 - Vista cranial do músculo diafragma do gato doméstico onde se evidenciam os tipos de ramificação e a distribuição dos nervos frênicos direito e esquerdo com suas respectivas freqüências de ocorrência. Legenda: a - hiato aórtico; o - hiato esofágico; $v$ - veia cava caudal; ct - centro tendíneo; pe - pilar esquerdo; pd - pilar direito; cmd - crus mediale dexter; cms - crus mediale sinister; fe - nervo frênico esquerdo; fd - nervo frênico direito.
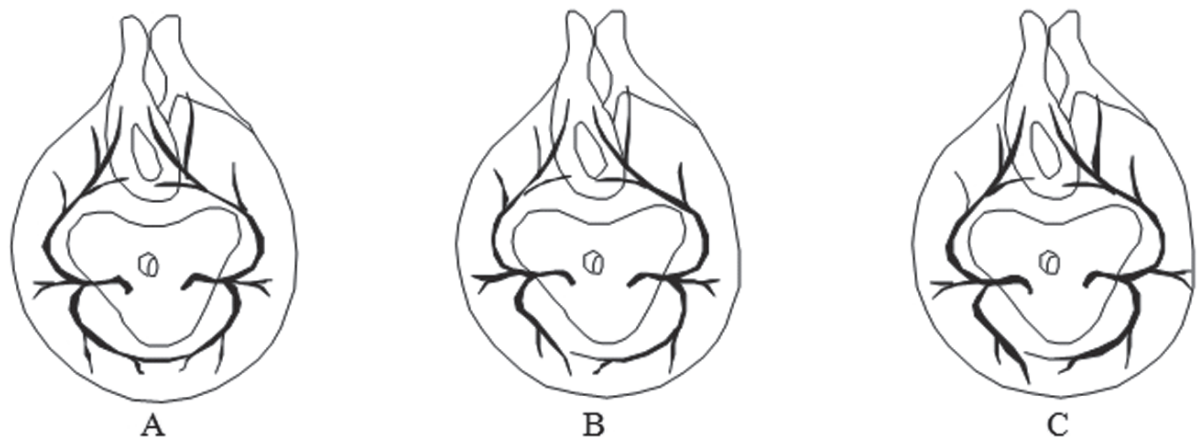

Figura 3 - Vista cranial do músculo diafragma do gato doméstico onde se evidencia o cruzamento de fibras nervosas provenientes do ramo ventral do nervo frênico esquerdo para o antímero direito. Em A se observa conexão entre fibras nervosas dos ramos ventrais dos nervos frênicos esquerdo e direito 
Constatou-se também a terminação em três ramos, dorsal, lateral e ventral dois casos à direita $(6,66 \%)$, concordando com os achados de Conde ${ }^{11}$ em cães $(15,0 \%)$, de Conde $^{8}$ em suínos $(5,0 \%)$ e distantes dos encontrados por Amorim Júnior, Bittencourt e Amorim ${ }^{9}$ no sagüi (46,6 \%). No antímero esquerdo observou-se essa trifurcação em 13 casos $(43,33 \%)$, assemelhando-se às descrições de Amorim Júnior, Bittencourt e Amorim ${ }^{9}$ no sagüi $(40,0 \%)$ e discordantes das observações de Conde ${ }^{8}$ em suínos (1,6 $\%)$ e de Santiago e Pereira ${ }^{12}$ em eqüinos $(6,7 \%)$.

A bifurcação em ramo dorsal e tronco ventrolateral foi evidenciada no gato doméstico três casos à direita $(10,0 \%)$ e um caso à esquerda $(3,33 \%)$, se assemelhando aos encontrados por Conde ${ }^{11}$ em cães $(15,0$ $\%)$ e por Santiago e Pereira ${ }^{12}$ em eqüinos (3,3 $\%$ dos casos à direita).

No atinente a esta terminação, Conde ${ }^{8}$ a descreveu nos suínos $(95,0 \%$ dos casos à direita e em 96,6 \% à esquerda), Santiago e Pereira $^{12}$ em eqüinos $(66,7 \%$ dos casos à esquerda), Miglino, Prada e Souza ${ }^{13}$ em búfalos (26,6\% à direita e $86,6 \%$ à esquerda) e Miglino, Souza e Didio ${ }^{14}$ em capivaras (73,3 $\%$ dos exemplares), informações estas que demonstram valores bem superiores aos encontrados no gato.

Observou-se que simetricamente os nervos frênicos do gato doméstico terminam se bifurcando em tronco dorsolateral e ramo ventral (36,66\%), arranjo este citado por Santiago e Pereira ${ }^{12}$ nos eqüinos $(26,7 \%$ ) e por Amorim Júnior, Bittencourt e Amorim 9 no sagüi $(33,3 \%)$, valores bem próximos dos encontrados no presente estudo.

Pode-se evidenciar a terminação em tronco dorsolateral e ventrolateral, exibida em dois casos $(6,66 \%)$ somente à direita. Tal arranjo não foi encontrado na literatura consultada.

A terminação do nervo frênico em tronco dorsolateral, ramos lateral e ventral, também foi observada no gato doméstico em um caso à esquerda (1,66\%). O ramo originado diretamente de um dos troncos de terminação do nervo frênico é denominado de acessório. Este é considerado um arranjo raro e foi descrito por Conde ${ }^{8,11}$ em suínos e cães, respectivamente.

Após a bifurcação ou trifurcação dos nervos frênicos, eles se distribuíam para a porção carnosa do músculo diafragma do gato doméstico sem emitir ramos para a sua porção tendínea, disposição esta citada por Miglino ${ }^{15}$ em caprinos e por Miglino, Prada e Souza ${ }^{13}$ em búfalos, nem tão pouco para a veia cava caudal, como relatado por Conde ${ }^{8,11}$ em suínos e cães, por Santiago e Pereira ${ }^{12}$ em eqüinos e por Miglino, Prada e Souza ${ }^{13}$ em búfalos. Entretanto, os achados deste estudo coincidem com os relatos de Amorim Júnior, Bittencourt e Amorim 9 para o sagüi.

Os ramos dorsais dos nervos frênicos inervaram a pars lumbalis $(73,33 \%$ à direita $\mathrm{e}$ $56,66 \%$ à esquerda) e a pars costalis $(13,33 \%$ à direita e 10,0\% à esquerda). O ramo dorsal direito supriu a crus mediale dexter do pilar direito em 100,0 \% dos casos, e o ramo dorsal esquerdo, inervou a crus mediale sinister do pilar direito além do pilar esquerdo em 100,0 \% dos casos.

Os ramos laterais inervaram à direita a pars lumbalis (23,33\%), a costalis $(96,66 \%) \mathrm{e}$ a sternalis $(3,33 \%)$. No antímero esquerdo, supriram somente a pars lumbalis $(33,33 \%) \mathrm{e}$ a costalis $(100,0 \%)$.

Os ramos ventrais distribuíram-se para a região ventral da pars costalis (46,66\% à direita e $43,33 \%$ à esquerda) e para a pars sternalis $(96,66 \%$ à direita $100,0 \%$ à esquerda).

Estas informações referentes à distribuição dos ramos dos nervos frênicos no músculo diafragma do gato doméstico são semelhantes às descritas por Amorim Júnior, Bittencourt e Amorim no sagüi, com pequenas variações em suas freqüências.

Não foram observadas conexões nervosas entre ramos homolaterais dos nervos frênicos conforme relataram Conde ${ }^{8,11}$ em suínos e cães, Santiago e Pereira $^{12}$ em eqüinos e Miglino, Prada e Souza ${ }^{13}$ em búfalos. Entretanto, evidenciouse conexão entre fibras em um dos exemplares em que ocorreu o cruzamento de fibras nervosas do ramo ventral do nervo 
frênico esquerdo para o antímero direito, concordando com os informes dos autores supracitados. De acordo com Amorim Júnior, Bittencourt e Amorim ${ }^{9}$ o sagüi não apresenta nenhuma destas observações.

\section{Conclusões}

O nervo frênico no gato doméstico se comporta de modo semelhante ao encontrado no sagüi e no cão em relação à sua ramificação, coincidindo com algumas observações encontradas em caprinos, em eqüinos e em bufalinos, entretanto, exibe uma distribuição distinta dessas espécies ocorrendo de maneira bem próxima à descrita no sagüi com exceção dos casos onde ocorre conexão de fibras entre os nervos frênicos contralaterais.

\title{
Ramification and distribution of the phrenic nerves in the muscle diaphragm of the domestic cat
}

\begin{abstract}
The ramification and the distribution of the phrenic nerves right and left had been studied in 30 muscles diaphragms of unknown breed adult domestic cats, 7 males and 23 females, and were fixed in $10 \%$ buffered formalin solution. After fixation and dissection, it was observed that the phrenic nerves ramified for the respective carnous parts of the muscle diaphragm, pars lumbalis, costalis and sternalis, and had finished in higher frequency in dorsolateral trunk and ventral branch $(63.33 \%)$. We observed the following arrangements: dorsal, ventral and lateral branches (25.0\%); dorsal branch and ventrolateral trunk (6.66 \%); dorsolateral and ventrolateral trunks (3.33\%); dorsolateral trunk, lateral and ventral branches $(1.66 \%)$. The phrenic nerves had distributed symmetrically in 11 samples $(36.66 \%)$, only showing the termination in dorsolateral trunk and ventral branch. The dorsal branches supplied pars lumbalis (73.33 \% to right and $56.66 \%$ to the left) and pars costalis $(13.33 \%$ to right and $10.0 \%$ to the left). The right dorsal branch supplied the crus mediale dexter of the right pillar $(100.0 \%)$ and the left dorsal branch supplied the crus mediale sinister of the right pillar and the left pillar $(100.0 \%)$. The lateral branches supply pars lumbalis $(23.33 \%$ to right and $33.33 \%$ to the left), pars costalis (96.66 \% to right and $100.0 \%$ to the left) and pars sternalis (3.33\% only to the right). The ventral branches supplied the ventral region of pars costalis (46.66 $\%$ to right and $43.33 \%$ to the left) and pars sternalis (96.66\% to right and $100.0 \%$ to the left). Four female animals $(13.33 \%$ had shown fibers crossing proceeding from the left ventral branch for right antimere had been that in one of these samples $(3.33 \%)$ occurred connection between the left ventral branch and the right.
\end{abstract}

Key words:

Ramification. Distribution

\section{Referências}

1 GETTY, R. Sisson/Grossman anatomia dos animais domésticos. 5. ed. Rio de Janeiro: Guanabara Koogan, 1986. v. 2, p. 1607-1617.

2 KÖNIG, H. E.; LIEBICH, H.G. Anatomia dos animais domésticos: texto e atlas colorido. Porto Alegre: Artmed, 2004. p. 252.

3 MONTAGNA, W. Anatomía comparada. 2. ed.
Barcelona: Omega, 1967. p. 151, 329-330.

4 SOUZA, W. M. et al. Considerações sobre os níveis de origem dos nervos frênicos em bovinos azebuados. Arquivos de Biologia e Tecnologia, Jaboticabal. v. 28, n. 4, p. 619-624, 1985.

5 TAMEGA, O. J. et al. Origem do nervo frênico no gato (Felis domestica). Arquivo da Escola de Veterinária da Universidade Federal de Minas Gerais, Belo Horizonte, v. 27, n. 2, p. 205-208, 1975. 
6 DYCE, K. M.; SACK, W. O.; WENSING, C. J. G. Tratado de anatomia veterinária. 3 . ed. Rio de Janeiro: Guanabara Koogan, 2004. p. 307-308.

7 MERENDINO, K. A.; et al. The intradiaphragmatic distribution of the phrenic nerve with particular reference to the placement of diaphragmatic incisions and controlled segmantal paralysis. Surgery, v. 39, p. 189198, 1956.

8 CONDE, R. Estudo anatômico com dados experimentais sobre a distribuição dos nervos frênicos no músculo diafragma de Sus scrofa domesticus, 1959. 110 f. Tese (Catedra) - Escola Superior de Veterinária, Universidade Rural do Estado de Minas Gerais. Belo Horizonte, 1959

9 AMORIM JÚNIOR, A. A.; BITTENCOURT, A. M.; AMORIM, M. J. A. A. L. Ramificação e distribuição dos nervos frênicos no diafragma de sagüi (Callithrix jacchus). Revista Brasileira de Ciências Morfológicas, v. 10 , n. 2 , p. $108-113,1993$

10 INTERNATIONAL COMMITTEE ON VETERINARY GROSS ANATOMICAL NOMENCLATURE. Nomina anatomica veterinaria. 4. ed. New York: World Association on Veterinary Anatomist, 1994. 198 p. (Together with nomina histologica, 2. ed. 1992 and nomina embryologica veterinaria, 1992).

11 CONDE, R. Estudo anatômico sobre a distribuição dos nervos frênicos no músculo diafragma de Canis familiaris, 1957. 36 f. Tese (Doutorado) - Escola Superior de Veterinária, Universidade Rural do Estado de Minas Gerais, Belo Horizonte. 1957.

12 SANTIGO, W.; PEREIRA, J. G. L. Contribuição ao estudo da inervação do diafragma em eqüinos da raça Puro Sangue Inglês. Revista da Faculdade de Medicina Veterinária e Zootecnia da USP, São Paulo, v. 20, n. 2, p. 143-153, 1983.

13 MIGLINO, M. A.; PRADA, I. L. S.; SOUZA, W. M. Ramificação e distribuição dos nervos frênicos direito e esquerdo no diafragma em búfalos da raça Jaffarabadi. Revista da Faculdade de Medicina Veterinária e Zootecnia da USP, v. 22, n. 2, p. 97- 106, 1985.

14 MIGLINO, M. A.; SOUZA, W. M.; DIDIO, L. J. A. Distribution of the phrenic nerves in the diaphragm of capybara (Hydrochoerus hydrochoerus). Archivio Italiano di Anatomia e di Embriologia, v. 93, n. 1-2, p. 109-118, 1988

15 MIGLINO, M. A. Divisão e distribuição dos nervos frênicos no músculo diafragma em caprinos, 1982. 40 f. Dissertação (Mestrado) - Faculdade de Medicina Veterinária e Zootecnia, Universidade de São Paulo, São Paulo 1982. 